\title{
More than just room temperature
}

\author{
Diluted magnetic semiconductors and oxides are interesting for fundamental science and applications \\ even without room-temperature ferromagnetism.
}

In April 2000, Tomasz Dietl and colleagues published a theoretical paper in Science that provided a model to explain the origin of ferromagnetism in (Ga,Mn)As, and used the same model to predict ferromagnetism in other semiconductors and oxides to above room temperature ${ }^{1}$. The results raised hopes of applications based on single elements combining the logic functionalities of semiconductors with the informationstorage capabilities of magnetic elements, and became a reference point for a large number of research groups who set off to find evidence of room-temperature ferromagnetism.

In this issue we explore the progress made during the past ten years regarding the origin of ferromagnetism in $(\mathrm{Ga}, \mathrm{Mn}) \mathrm{As}-$ widely considered the prototype diluted magnetic semiconductor (DMS) - and the search for high Curie temperatures $\left(T_{\mathrm{C}}\right)$ in other systems.

The field of DMSs originated in the late 1970s and developed in the 1980s with work on II-VI semiconductors, when it was realized that a small percentage of magnetic impurities would not deteriorate the optical and electronic transport properties of the host, and would simultaneously introduce large magnetic field effects ${ }^{2}$. (Ga,Mn)As took centre stage in 1996, when Hideo Ohno's group demonstrated ferromagnetic order in this compound with a $T_{\mathrm{C}}$ of $110 \mathrm{~K}$ (ref. 3) which, with improvements in the control of the material quality, has subsequently risen to $190 \mathrm{~K}$ (ref. 4).

Dietl's model was based on that introduced long before by Zener to explain ferromagnetism in metals ${ }^{5}$, according to which the ferromagnetic order among the spins of isolated Mn atoms is mediated by holes. The role of the carriers is a crucial aspect of the model, as it implies that the magnetic properties can be tuned by changing the carrier density in the materials, for example through external gating, as was demonstrated soon after ${ }^{6}$.

Still, even after ten years of intense research, consensus over the origin of ferromagnetism in (Ga,Mn)As has not been reached $^{7,8}$. The Zener model explains most of the prominent features, but some of the fundamental aspects are still controversial. For example, there is considerable debate about whether the impurity band

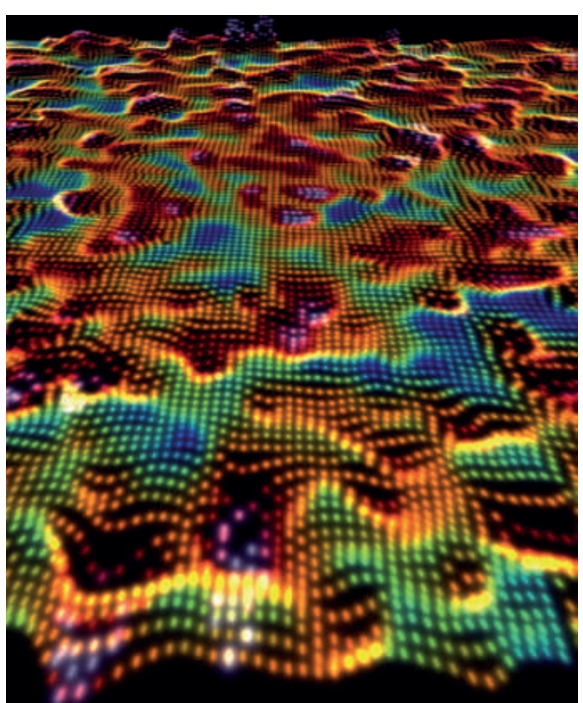

Electronic states generated by hole doping in ( $G a, M n)$ As show the spatial structure anticipated for electrons near the metal-insulator transition ${ }^{10}$.

associated with the holes is merged with the valence band or is decoupled from it. This technical aspect is essential to understand ferromagnetism in this material. Dietl himself believes in the first scenario ${ }^{7}$, but the alternative one is supported by several pieces of evidence, like the very recent result of resonant tunnelling experiments in (Ga,Mn)As quantum wells 9 .

Despite the lack of a comprehensive picture, this material can be considered as a model system for studying the carrier interaction with spins, and the experimental and theoretical tools developed in the past few years provide more insight into the physics of this system. For example, recent scanning tunnelling microscopy experiments highlight the importance of carrier correlations ${ }^{10}$ (see image).

Numerous observations of ferromagnetism at or above room temperature have been reported, particularly in oxides. Unfortunately, as Scott Chambers stresses in his Interview, the results were mostly irreproducible, and often likely due to spurious effects ${ }^{11}$. Many scientists are now convinced that Dietl's predictions on room-temperature ferromagnetism are not realizable, also due to unrealistic assumptions ${ }^{12}$. Nevertheless, the intense work the paper generated has been fruitful in other ways. The strive for improving the quality of the materials has led to high control of the growth processes and to the development of dedicated characterization techniques, primarily based on electron microscopy or X-rays. As Dietl explains in the second part of his Review ${ }^{7}$, these techniques make it now possible to visualize the formation of magnetic ion clusters and envisage ways to control it. This may one day be used to realize structures in which the magnetic properties of the semiconductors are controlled by purposefully designed magnetic clusters, rather than isolated ions.

With $T_{\mathrm{C}}$ of the prototype compound still at $190 \mathrm{~K}$ the prospects for applications may seem very slim indeed. However, as Hideo Ohno describes in his Commentary, ferromagnetic semiconductors, (Ga,Mn)As in particular, can provide fundamental insight into phenomena that when present in other types of materials - like some ferromagnetic metals - are highly relevant for spintronic applications ${ }^{13}$. These include the control of ferromagnetic properties by electric gating and the motion of magnetic domain walls that has been proposed as the basis of the next generation of storage devices ${ }^{14}$.

All in all, ferromagnetism in semiconductors and oxides remains a controversial issue, and whether the high Curie temperatures once predicted will ever be reached remains an open question. On the other hand, these materials have already generated interesting physics and served as model systems for the study of phenomena of interest for applications, and they will keep doing so for years to come.

\footnotetext{
References

1. Dietl, T., Ohno, H., Matsukura, F., Cibert, J. \& Ferrand, D. Science 287, 1019-1022 (2000).

2. Furdyna, J. J. App. Phys. 64, R29-R56 (1988).

3. Ohno, H. et al. Appl. Phys. Lett. 69, 363-365 (1996).

4. Wang, M. et al. Appl. Phys. Lett. 93, 132103 (2008).

5. Zener, C. Phys. Rev. 81, 440-444 (1951)

6. Ohno, H. et al. Nature 408, 944-946 (2000).

7. Dietl, T. Nature Mater. 9, 965-974 (2010).

8. Interview with N. Samarth. Nature Mater. 9, 955-956 (2010).

9. Ohya, S., Takata, K. \& Tanaka, M. Preprint available at http://arxiv.org/abs/1009.2235 (2010).

10. Richardella, A. et al. Science 327, 665-669 (2010).

11. Interview with S. Chambers. Nature Mater. 9, 956-957 (2010).

12. Zunger, A., Lany, S. \& Raebiger, H. Physics 3, 53 (2010).

13. Ohno, H. Nature Mater. 9, 952-954 (2010).

14. Parkin, S. S. P., Hayashi, M. \& Thomas, L. Science 320, 190-194 (2008).
} 